\title{
Occlusal analysis and management of a patient with headache and temporomandibular joint sound: A case
} report

\author{
Kengo Torii* \\ Department of Comprehensive Dental Care Unit, School of Life Dentistry, Nippon Dental University, Tokyo, Japan
}

\begin{abstract}
Although headaches attributed to masticatory myofascial pain have been reported, to our knowledge, the treatment of headache with occlusal position correcting therapy has not been reported. Occlusal improvement was achieved using the occlusal position correcting therapy in a patient with headache and temporomandibular joint sound. Occlusal analysis was performed by mounting the dental models on an articulator and identifying a premature occlusal contact on the right first premolars and a deviation of the habitual occlusal position from the muscular reference position. Occlusal modification was performed to coincide the habitual occlusal position with the muscular reference position. After the completion of occlusal equilibration, the chronic headache immediately became an episodic headache, and after the fourth month, the headache almost disappeared. The headache was believed to be caused by myofascial pain of the lateral and medial pterygoid muscles produced with the occlusal discrepancy between the habitual occlusal position and the muscular position. The headache was considered a referred pain from both the lateral and medial pterygoid muscles.
\end{abstract}

\section{Introduction}

Some studies have reported on the relationship between temporomandibular disorders (TMD) and headaches [1-6]. However, to our knowledge, limited information is available on the relationship between the headaches and dental occlusion [7]. Temporomandibular joint (TMJ) sound associated with the symptoms of TMD is reportedly related to the occlusal discrepancy between the habitual occlusal position (HOP) and the bite plate-induced occlusal position (BPOP) [8]. BPOP refers to the physiological muscular position. Some masticatory muscle tension might be caused by the occlusal discrepancy between the HOP and the muscular position, resulting in tension-type headache [9]. The present case study might help understand one of the mechanisms underlying tension-type headache.

\section{Case presentation}

A 31-year-old woman presented with the chief complaint of left TMJ sound. She had been experiencing a chronic headache for six years. She reported having taken days off work because of the headache. She was diagnosed as having migraine by a neurosurgeon and was treated with naratriptan hydrochloride and lomerizine hydrochloride. She took lomerizine twice daily and took naratriptan when she had a headache. She took naratriptan once every two days; however, there was no reduction in the headache. The patient's medical history was unremarkable, except the bilateral headache. She reported left TMJ sound during mouth opening. Mouth opening was $42 \mathrm{~mm}$, and the opening path deviated laterally to the left. There was no TMJ tenderness; however, tenderness of the lateral and medial pterygoid muscles on both the sides and the left trapezius on palpation were observed. Twenty-eight teeth were present (Figure 1). The TMJs bilaterally appeared normal on the computed tomography images taken in the HOP at the time of the
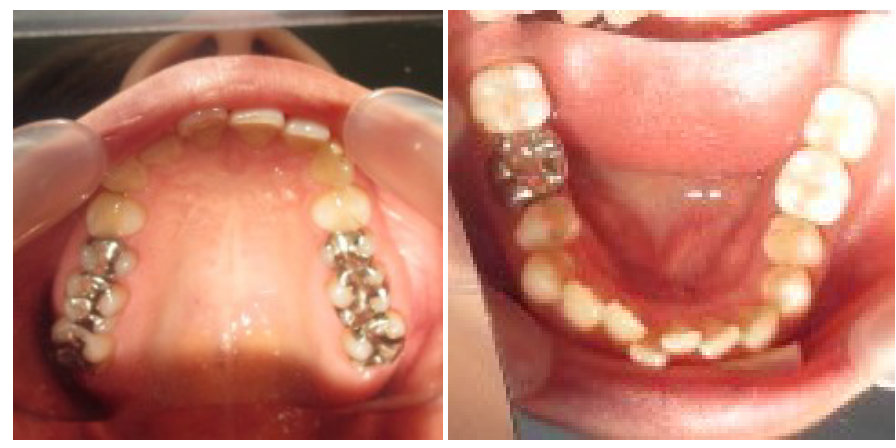

Figure 1. Upper and lower dental arches at the initial consultation

initial consultation (Figure 2). At the initial visit, a HOP record was obtained using a vinyl polysiloxane bite registration material (Exabite, GC, Tokyo, Japan), while the patient was seated upright with her jaw voluntarily closed. Subsequently, upper and lower jaw impressions were obtained, and the dental models were fabricated. An anterior flat bite plate was fabricated on the upper cast using a self-curing acrylic resin (Ortho-fast, GC, Tokyo, Japan). The patient was instructed to record the dose of medicine taken each day, and the headache intensity was

${ }^{\star}$ Correspondence to: Kengo Torii, Department of Comprehensive Dental Care Unit, School of Life Dentistry, Nippon Dental University, Tokyo, Japan, Tel:054247-2966; E-mail: wbs89508@mail.wbs.ne.jp

Key words: myofascial pain, habitual occlusal position, muscular reference position, occlusal discrepancy

Received: May 11, 2020; Accepted: May 22, 2020; Published: May 26, 2020 
scored on a 10-point Visual Analogue Scale (VAS). At the second visit, she wore the bite plate for $5 \mathrm{~min}$, and the BPOP record was obtained, using the same material as that used for obtaining the HOP record (Figure 3). A BPOP wax record was obtained using the registration wax material (Bite wafer, Kerr USA, Romulus, MI, U.S.A.) using the method described previously. The upper and lower models were mounted on an articulator with the BPOP wax record. A premature occlusal contact was recognized on the right first premolars (Figure 4). To examine the difference between HOP and BPOP, three dimensional measurements were performed with the modified articulator using previous records (Figure 5). Her HOP was deviated antero-right laterally from the BPOP, the physiological muscular position (Figure 6). The shifts ranged from $0.8 \mathrm{~mm}$ to $2.0 \mathrm{~mm}$. Occlusal adjustment was performed on the dental models mounted on the articulator until the posterior teeth bilaterally made contact as per the occlusal position correcting therapy (Figure 7) [10]. Subsequently, the occlusal adjustments in the mouth were performed following afore mentioned procedure. The adjustments were performed at five visits. The tenderness of the left trapezius and the lateral and medial pterygoid muscles on both the sides disappeared, and the TMJ sound on the left side also disappeared after completion of the adjustment. The condyle on the right side appeared to be posterior in the glenoid fossae (Figure 8). The patient was asked to discontinue lomerizine.

The number of doses of naratriptan reduced considerably after occlusal adjustments, and the chronic headache immediately turned into an episodic headache. After the fourth month, the headache almost disappeared (Figure 9) [11].

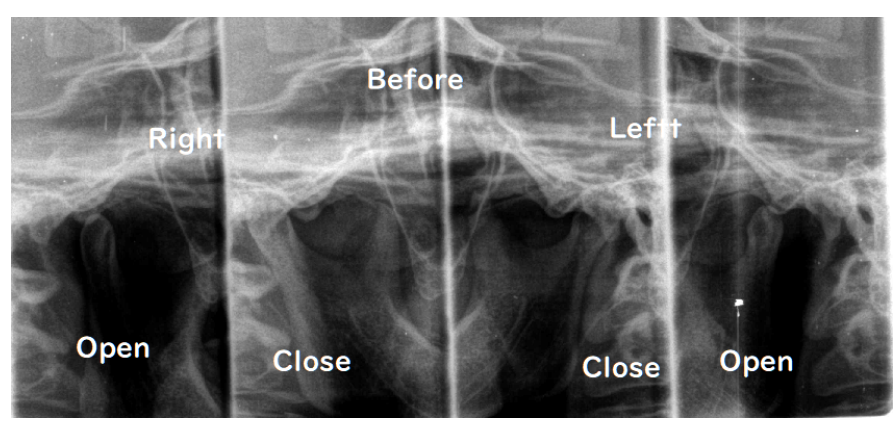

Figure 2. Bilateral tomographic images of TMJ in the open and closed positions

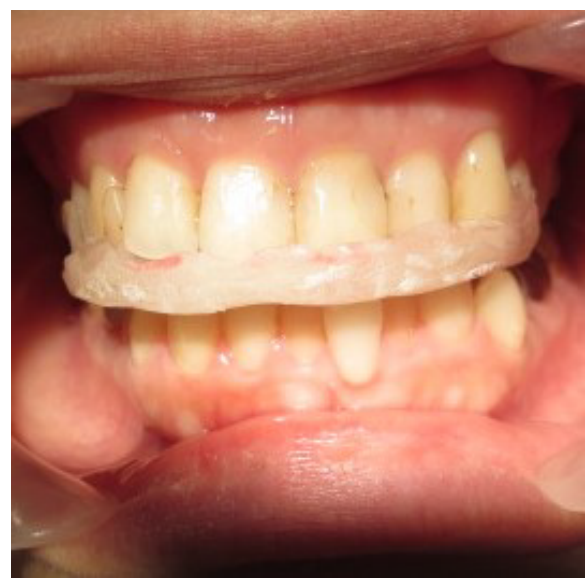

Figure 3. Wearing the bite plate

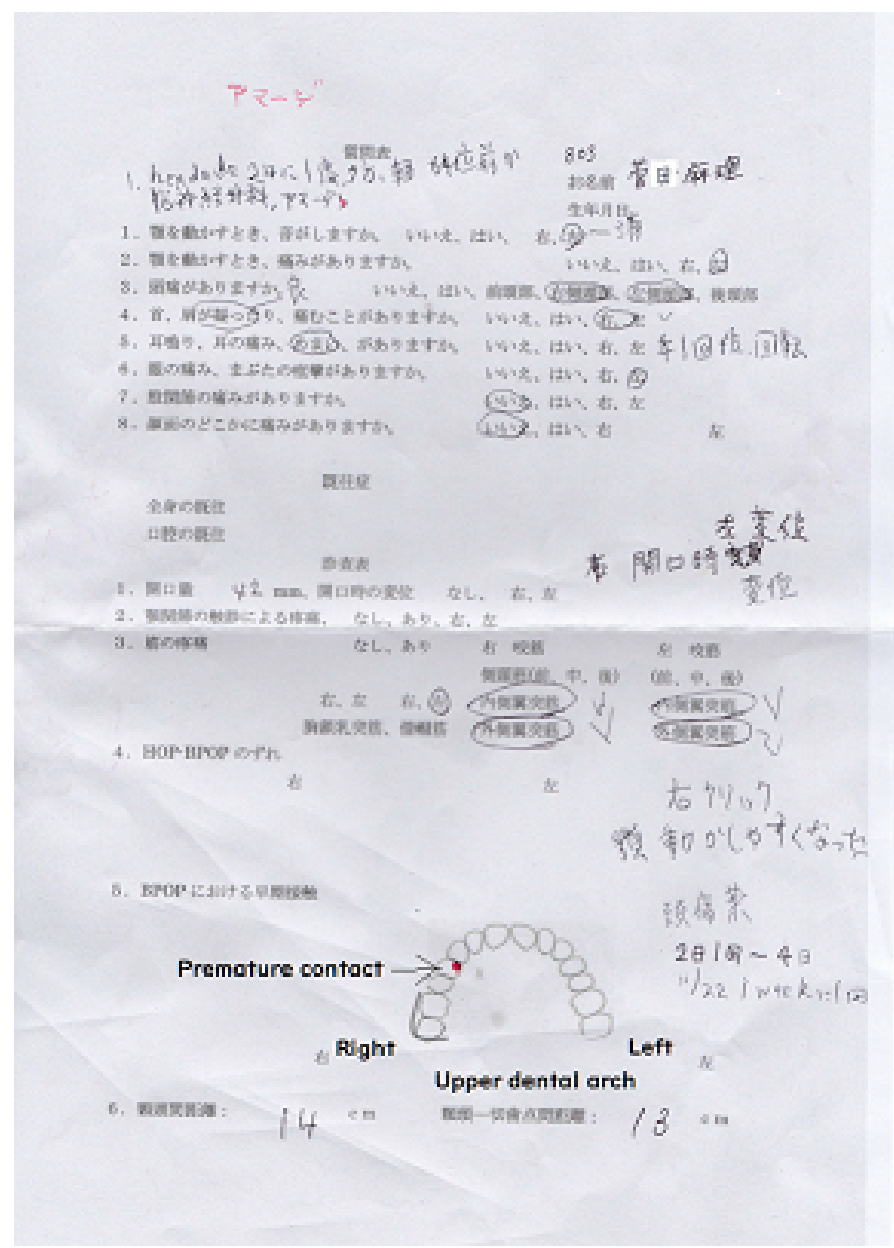

Figure 4. Examination record

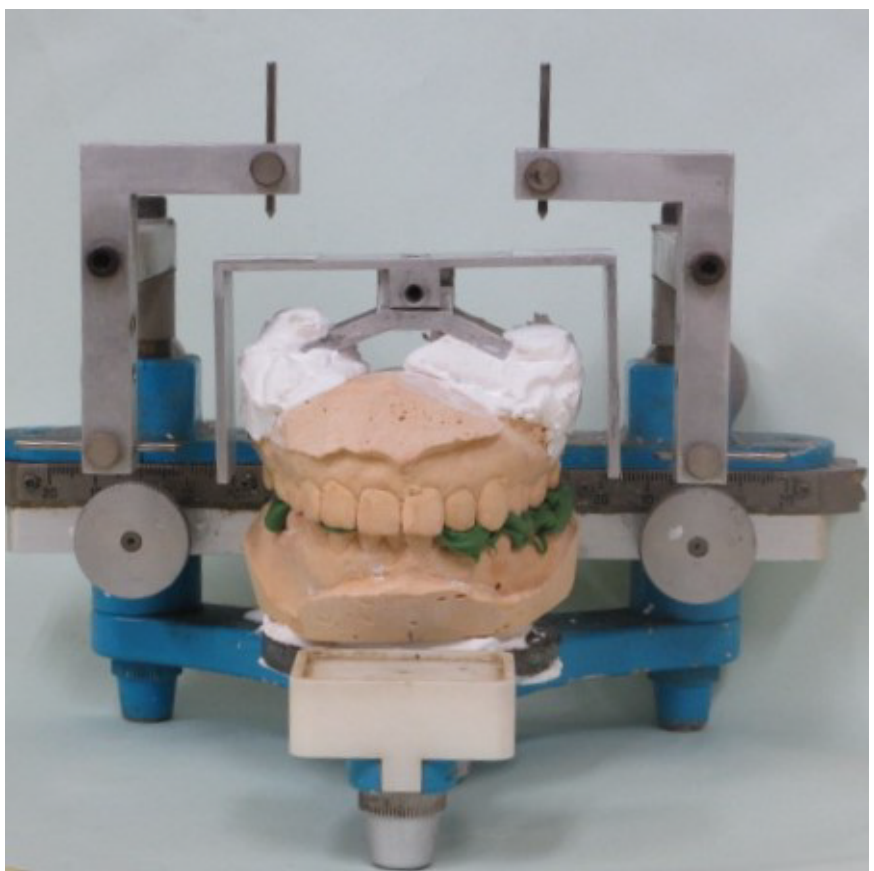

Figure 5. Apparatus added to an articulator for three-dimensional occlusal analysis 


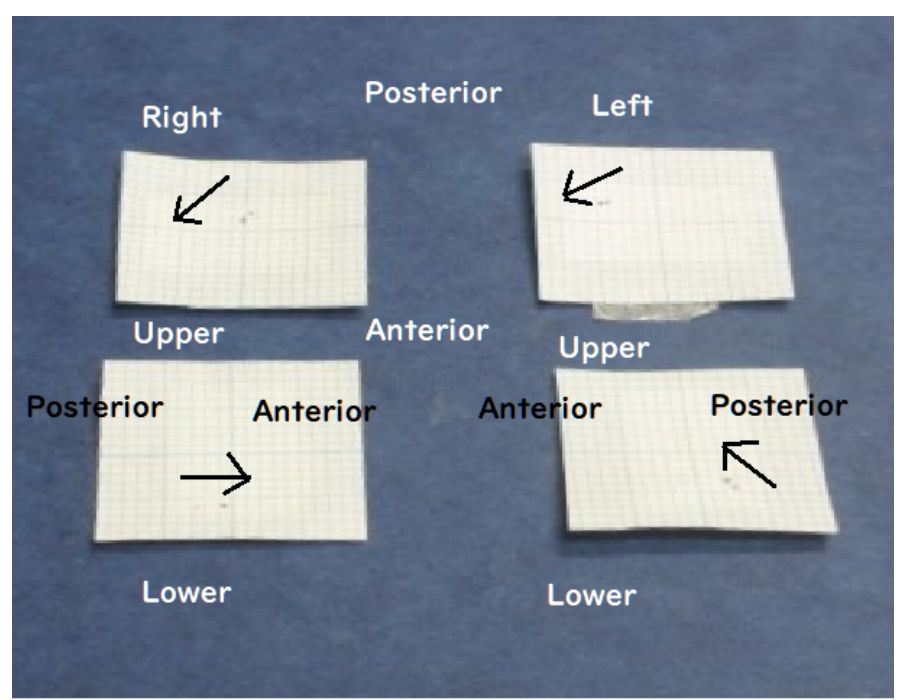

Figure 6. The HOP and BPOP records. The arrows indicate the shifts from the BPOP to the HOP

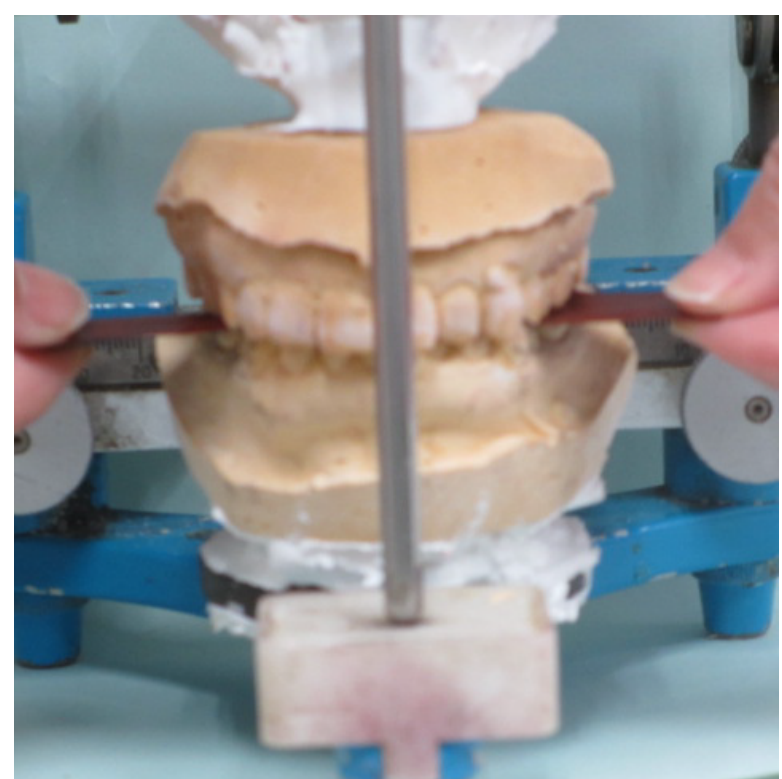

Figure 7. Confirmation of occlusal contacts on both the sides in the muscular contact position

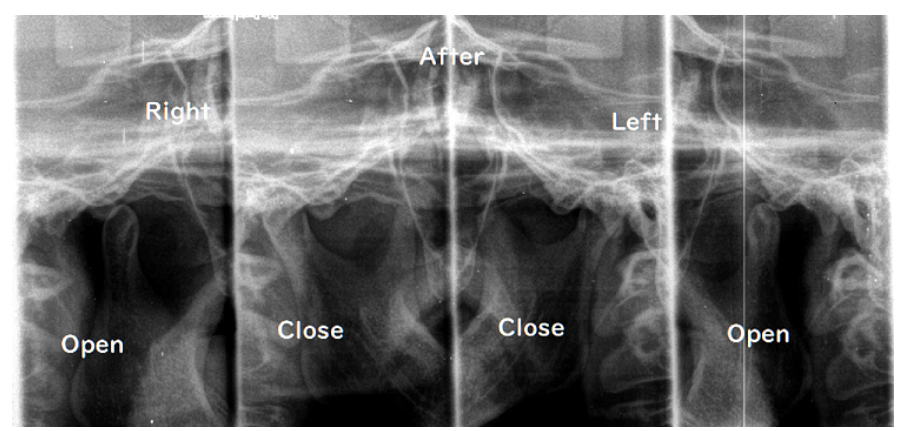

Figure 8. Bilateral tomographic images of TMJ in the open and closed positions after completion of occlusal equilibration

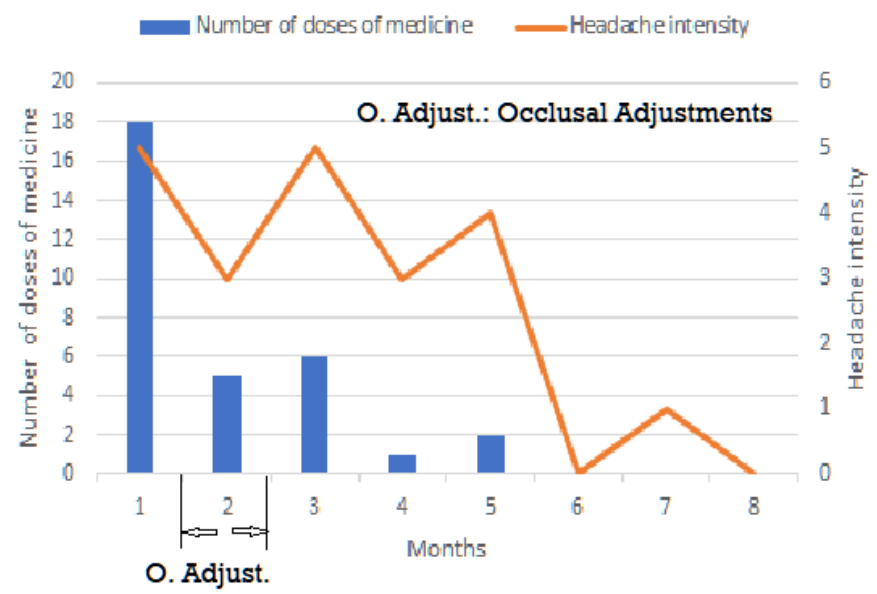

Figure 9. Monthly changes in the number of doses of headache medicine and the intensity of headache

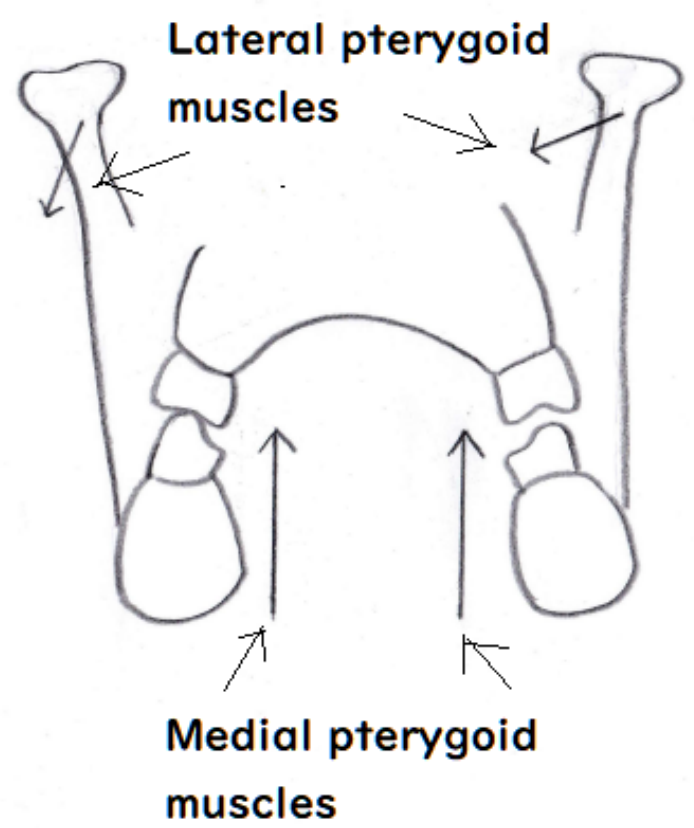

Figure 10. Additional muscle activities required to move from BPOP to HOP

\section{Discussion}

Although headache attributed to TMD has been managed via counseling, occlusal appliance, or physical therapy, the outcomes have been temporal $[1,6]$. Costa et al. reported that the main clinical features of headache attributed to masticatory myofascial pain were long duration and frontotemporal bilateral location. Moreover, they described that the mean ( \pm standard deviation $[S D]$ ) baseline VAS was $7.6( \pm 2.2)$ for group 1 (only counseling) and $6.5( \pm 1.6)$ for group 2 (counseling and an occlusal appliance; final values were $3.1([ \pm 2.2][\mathrm{P}<0.001])$ and $2.5( \pm$ 2.3) $(\mathrm{P}<0.001)$, respectively [1]. Hara et al. reported that the headache reduced during TMD treatment, and the improvement was temporally related to the amelioration of TMD symptoms [6]. In the present case, the disappearance of TMJ sound after occlusal treatment was also related to reduction in headache. Torii and Chiwata reported that 5 of 
13 headache patients did not experience any further headaches after occlusal adjustment using the BPOP as a reference position [7]. In the present case, the move from the BPOP to HOP means that it requires extra muscle activity of the lateral and medial pterygoid muscles (Figure 10). These muscles are smaller than the masseter or temporal muscle. Therefore, these movements that are repeated in mastication would exceed the activity capacity of these muscles and cause myofascial pain. The patient cannot feel the myofascial pain of the lateral and medial pterygoid muscles; therefore, the patient's body emits the referred pain on the bilateral location of the head as an alert. Las-Peñas described that sensitization of the nociceptive pain pathways in the central nervous system due to prolonged nociceptive stimuli from myofascial trigger points appear to be responsible for the conversion of episodic to chronic tension-type headache [9]. In the present case, immediately after the completion of occlusal adjustment, the number of doses of naratriptan and the intensity of headache were increased, potentially owing to the discontinuation of lomerizine. Lomerizine suppresses the contraction of blood vessels and the increase of blood flow to the brain; therefore, it is considered that its discontinuation decreased blood flow to the brain that caused a severe headache as a reaction. In the present case, the headache did not disappear immediately after the completion of occlusal treatment, presumably because the path of referred pain formed in the brain persisted. The headache in the present case was not judged to be a migraine, but a tension-type headache.

\section{Acknowledgment}

Written consent was obtained from the patient prior to the publication of this study.

The author would like to thank Mrs. Yuki Chiba who drew the illustration.

\section{References}

1. Costa YM, Porporatti AL, Stuginski-Barbosa J, Bonjardim LR, Speciali JG, et al. (2015) Headache attributed to masticatory myofascial pain: Clinical features and management outcomes. J Oral Facial Pain Headache 29: 323-330.

2. Tchivieva IE, Ohrbach R, Fillingim RB, Greenspan JD, Maixner W, et al. (2017) Temporal change in headache and its contribution to the risk of developing first-onset temporomandibular disorder in the orofacial pain: Prospective evaluation and risk assessment (OPPERA) study. Pain 158: 120-129.

3. Watanabe N, Shimohira M (2005) The secondary headache in dentistry. Nihon Rinsho 63: $1777-1785$.

4. Tomaz-Morais JF, Lucena LB, Mota IA, Pereira AK, Lucena BT, et al. (2015) Temporomandibular disorder is more prevalent among patients with primary headaches in a tertiary outpatient clinic. Arg Neuropsiquiatr 73: 913-917.

5. Vivaldi D, Di Gioisa M, Tchivileva IE, Jay GW, Slade GD, et al. (2018) Headache attributed to TMD is associated with the presence of comorbid bodily pain: A casecontrol study. Headache 58: 1593-1600.

6. Hara K, Shinozaki T, Okada-Ogawa A, Matsukawa Y, Dezawa K, et al. (2016) Headache attributed to temporomandibular disorders and masticatory myofascial pain. J Oral Sci 58: 195-204.

7. Torii K, Chiwata I (2010) Occlusal adjustment using the bite plate-induced occlusal position as a reference position for temporomandibular disorders: A pilot study. Head \& Face Medicine 6: 5 .

8. Torii K, Chiwata I (2005) Relationship between habitual occlusal position and fat bite plane-induced occlusal position in volunteers with and without temporomandibular joint sounds. J Craniomandib Pract 23: 16-21.

9. Fernández-de-Las-Peñas C (2015) Myofascial head pain. Curr Pain Headache Rep 19: 28.

10. Torii K (2018) Occlusal position correcting therapy for temporomandibular disorders. EC Dental Science 17: 168-176.

11. Syed Ahmed (2019) Muscle contraction tension headache: eMedicine Neurology.

Copyright: (C2020 Torii K. This is an open-access article distributed under the terms of the Creative Commons Attribution License, which permits unrestricted use, distribution, and reproduction in any medium, provided the original author and source are credited. 